\title{
Inverses of generators of nonanalytic semigroups
}

\author{
by
}

\author{
Ralph DeLaubenfels (Columbus, OH)
}

\begin{abstract}
Suppose $A$ is an injective linear operator on a Banach space that generates a uniformly bounded strongly continuous semigroup $\left\{e^{t A}\right\}_{t \geq 0}$. It is shown that $A^{-1}$ generates an $O(1+\tau) A(1-A)^{-1}$-regularized semigroup. Several equivalences for $A^{-1}$ generating a strongly continuous semigroup are given. These are used to generate sufficient conditions on the growth of $\left\{e^{t A}\right\}_{t \geq 0}$, on subspaces, for $A^{-1}$ generating a strongly continuous semigroup, and to show that the inverse of $-d / d x$ on the closure of its image in $L^{1}([0, \infty))$ does not generate a strongly continuous semigroup. We also show that, for $k$ a natural number, if $\left\{e^{t A}\right\}_{t \geq 0}$ is exponentially stable, then $\left\|e^{\tau A^{-1}} x\right\|=O\left(\tau^{1 / 4-k / 2}\right)$ for $x \in \mathcal{D}\left(A^{k}\right)$.
\end{abstract}

I. Introduction. Let $A$ be an injective linear operator on a Banach space. When $A$ has dense image and generates a uniformly bounded strongly continuous analytic semigroup, it is well known that $A^{-1}$ also generates such a semigroup (see [Gor-M] and [d1]). The same equivalence is not true with "analytic" removed; in $[\mathrm{Z} 1]$, [Gom-Z-T], and $[\mathrm{K}]$ it is shown that there exists an injective linear operator with dense image on a Banach space that generates a uniformly bounded strongly continuous semigroup whose inverse does not generate a strongly continuous semigroup. In [Gom-Z-T] this is done on $\ell^{p}, p \in(1,2) \cup(2, \infty)$. The question of $A^{-1}$ generating a strongly continuous semigroup when $A$ generates a uniformly bounded strongly continuous semigroup on a Hilbert space remains open (but see [Gom-Z-T] for a strong negative step in this direction; see also [Z2]).

These negative results lead naturally to the following questions.

(1) What can be said about inverses of generators of uniformly bounded semigroups?

(2) What (relatively simple) properties are equivalent to both $A$ and $A^{-1}$ generating strongly continuous semigroups?

2000 Mathematics Subject Classification: Primary 47A60, 47D03; Secondary 47D60, $47 \mathrm{D} 62$.

Key words and phrases: strongly continuous semigroups of operators, functional calculus, operational calculus, regularized semigroups, $C$-semigroups, integrated semigroups. 
(3) What (relatively simple) sufficient conditions for both $A$ and $A^{-1}$ generating strongly continuous semigroups can be derived?

(4) More generally, what is the relationship between (possibly not strongly continuous) semigroups generated by $A$ and (possibly not strongly continuous) semigroups generated by $A^{-1}$ ?

When $A$ generates a uniformly bounded strongly continuous semigroup $\left\{e^{t A}\right\}_{t \geq 0}$, nothing could be more natural than to take the following Laplace transform (see $[\mathrm{Z1},(10)]$ and $[\mathrm{D}]$ ):

$$
\int_{0}^{\infty} e^{-s t} \tau G(t \tau) d t=1-e^{-\tau / s} \quad(\tau \geq 0),
$$

where $G(t) \equiv(1 / \sqrt{t}) J_{1}(2 \sqrt{t}), J_{1}$ the Bessel function of the first kind of the first order, and replace the nonnegative real number $s$ with the operator $-A$, to get, at least formally, a construction of a semigroup $\left\{e^{\tau A^{-1}}\right\}_{\tau \geq 0}$ generated by $A^{-1}$ :

$$
e^{\tau A^{-1}} x=x-\int_{0}^{\infty}\left(e^{t A} x\right) \tau G(t \tau) d t \quad(x \in X, \tau \geq 0) .
$$

This fails to define a strongly continuous semigroup, in general, precisely because $G$ is not in $L^{1}([0, \infty))$. See [Z1, Lemma 3.2] for the validity of (1.2) when $\left\{e^{t A}\right\}_{t \geq 0}$ is exponentially stable.

However, $G$ becomes an $L^{1}$ function after convolution with $e^{-t}-\delta_{0}(t)$, whose Laplace transform is $(1+s)^{-1}-1=-s(1+s)^{-1}$. Thus it is natural to regularize $e^{\tau A^{-1}}$ with $A(1-A)^{-1}$; we show (Theorem 3.3) that $A^{-1}$ generates an $O(1+\tau) A(1-A)^{-1}$-regularized semigroup (see Definition 2.2) $\left\{e^{\tau A^{-1}} A(1-A)^{-1}\right\}_{\tau \geq 0}$ whenever $A$ is injective and generates a uniformly bounded strongly continuous semigroup. We also recover the intuitive functional calculus representation (1.2), for $x \in \operatorname{Im}(A)$, the image of $A$; this will be fundamental to Section IV. See [P-Z, Theorem 3.3] for a different construction of a once-integrated semigroup (see Definition 2.1) generated by $A^{-1}$.

Section III also shows that, when $\left\{e^{t A}\right\}_{t \geq 0}$ is exponentially stable, then $\left\|e^{\tau A^{-1}} x\right\|=O\left(\tau^{1 / 4-k / 2}\right)$ for $x \in \mathcal{D}\left(A^{k}\right), k \in \mathbb{N}$ (Theorem 3.9). For $k=1$, this has been shown in [Z1, Theorem 3.3]. Propositions 3.10 and 3.11 give similar results under the weaker hypothesis that the resolvent of $A$ be bounded in a right half-plane containing the imaginary axis.

In Section IV, when $A$ is an injective generator of a uniformly bounded strongly continuous semigroup on a Banach space $X$, we use Theorem 3.3 to construct many conditions equivalent to $A^{-1}$ generating a strongly continuous semigroup (Theorem 4.3). Most of these characterizations are in terms of (1.2) above; for example, $A^{-1}$ generates a strongly continuous semigroup 
if and only if $\operatorname{Im}(A)$ is dense and

$$
S(\tau) y \equiv \int_{0}^{\infty}\left(e^{t A} y\right) \tau G(t \tau) d t \quad(y \in \operatorname{Im}(A), \tau \geq 0)
$$

(convergence of the integral guaranteed by Theorem 3.3) is a family of bounded operators from $\operatorname{Im}(A)$ to $X$ locally bounded in $\tau$ if and only if $S(\tau)$ leaves $\operatorname{Im}(A)$ invariant, with $\left(1-A^{-1}\right) S(\tau) A(1-A)^{-1}$ defining a family of bounded operators on $X$ locally bounded in $\tau$. The semigroup generated by $A^{-1}$ then has the form

$$
e^{\tau A^{-1}} x=x+\left(1-A^{-1}\right) S(\tau) A(1-A)^{-1} x
$$

for $x \in X$ and $\tau \geq 0$. Another equivalence of Theorem 4.3 is the following curiosity: $A^{-1}$ generating a $(1-A)^{-2}$-regularized semigroup is equivalent to $A^{-1}$ generating a strongly continuous semigroup.

Theorem 4.3 is applied to the prototype of uniformly bounded strongly continuous semigroup generators, the operator $A \equiv-d / d x$ on $L^{1}([0, \infty))$. By restricting $A$ to the closure of its image, we have an injective operator with dense image that generates a uniformly bounded strongly continuous semigroup. We use Theorem 4.3 to show that the inverse of this restriction does not generate a strongly continuous semigroup (Example 4.4).

Section IV then uses the equivalences in Theorem 4.3 to generate sufficient conditions for $A^{-1}$ generating a strongly continuous semigroup. These begin with, again, the representation (1.2): it is sufficient that the integral in (1.2) converge, after being regularized by $(1-A)^{-2}$, for all $\tau \geq 0$, and define a strongly continuous family of bounded operators (Corollary 4.5). Other sufficient conditions, involving the rate of growth of $\left\|e^{t A}\right\|$ or $\left\|\int_{0}^{t} e^{s A} d s\right\|$, on subspaces, appear in Theorems 4.7 and 4.13 and Proposition 4.11, sometimes giving other integral representations besides (1.2) for $e^{\tau A^{-1}}$. Proposition 4.10 gives similar sufficient conditions for $\left\|e^{\tau A^{-1}} x\right\|$ to be $O\left(\tau^{-1 / 4}\right)$, generalizing [Z1, Theorem 3.3]. Theorem 4.13 actually does not require that $A$ generate a strongly continuous semigroup; it is sufficient that $A$ generate a once-integrated semigroup (see Definition 2.1) that is $O\left(t^{r}\right)$, as $t$ goes to infinity, for some $r<1 / 4$, in order that $A^{-1}$ generate a strongly continuous semigroup. See [Gom2] for sufficient conditions on the resolvent of $A$, in order that $A^{-1}$ generate a uniformly bounded strongly continuous semigroup.

Theorems 3.9 and 4.13 hint at a possible general duality between $A$ and $A^{-1}$. Loosely speaking, regularity of $e^{t A}$ seems to be equivalent to decay of $e^{\tau A^{-1}}$ (hence the converse).

There is also an interesting relationship between both $A$ and $A^{-1}$ generating uniformly bounded strongly continuous semigroups, and the Cayley transform of $A$; see [Gom1] and [Gom-Z]. 
This paper uses unbounded extensions of both the Hille-Phillips functional calculus (see (2.4)), as in (1.2), and the Riesz-Dunford holomorphic functional calculus. These are described in Section II, after the definitions of integrated and regularized semigroups.

We conclude with some open questions.

See [Z1, Introduction] for an interesting description of many applications of $A^{-1}$ being a generator to control theory and numerical analysis.

Throughout this paper, all operators are linear, on a Banach space, $X$, and $A$ is an injective operator from a subspace of $X, \mathcal{D}(A)$, into $X$. Also denote by $\varrho(G)$ the resolvent set of the operator $G$, by $\operatorname{Im}(G)$ its image, and by $B(X)$ the space of all bounded operators from $X$ into itself. When an operator $G$ generates a strongly continuous semigroup, it will be denoted $\left\{e^{t G}\right\}_{t \geq 0}$. More generally, if $G$ generates a $C$-regularized semigroup $\{W(t)\}_{t \geq 0}$ (see Definition 2.2), then $e^{t G} \equiv C^{-1} W(t)$, for $t \geq 0$, defines a semigroup of possibly unbounded operators. For a possibly vector-valued function $H$, " $\int_{0}^{\infty} H(t) d t$ " will be shorthand for " $\lim _{N \rightarrow \infty} \int_{0}^{N} H(t) d t$ exists and equals $\int_{0}^{\infty} H(t) d t . "$

II. Preliminaries. Basic material on strongly continuous semigroups may be found in [Gol], [H-P], [N], [E-N], [Pa], and [vC]. A semigroup $\left\{e^{t A}\right\}_{t \geq 0}$ is exponentially stable if its norm is bounded above by $M e^{-\varepsilon t}$ for some $M$, $\varepsilon>0$.

First we need to describe two generalizations of strongly continuous semigroups, and their relationship to the abstract Cauchy problem

$$
\frac{d}{d t} u(t, x)=A(u(t, x)) \quad(t \geq 0), \quad u(0, x)=x \in X .
$$

Definition 2.1. For $n$ a nonnegative integer, a strongly continuous family $\{S(t)\}_{t \geq 0}$ of operators is an exponentially bounded $n$-times integrated semigroup generated by $A$ if, for some real $\omega,(\omega, \infty) \subseteq \varrho(A)$ and

$$
(\lambda-A)^{-1} x=\lambda^{n} \int_{0}^{\infty} e^{-\lambda t} S(t) x d t
$$

for all $\lambda>\omega$ and $x \in X$. Generation of an $n$-times integrated semigroup corresponds to (ACP) having a unique mild solution $u(t, x)=(d / d t)^{n} S(t) x$ for all $x \in \mathcal{D}\left(A^{n}\right)$. A 0 -times integrated semigroup is a strongly continuous semigroup. See $[\mathrm{A}]$ for basic information on integrated semigroups.

Definition 2.2. For $C$ a bounded, injective operator, a $C$-regularized semigroup is a strongly continuous family $\{W(t)\}_{t \geq 0}$ of operators such that $W(0)=C$ and, for $s, t \geq 0$,

$$
W(t) W(s)=C W(t+s) .
$$


The generator of $\{W(t)\}_{t \geq 0}$ is an operator $A$ such that

$$
A x=C^{-1}\left(\left.\frac{d}{d t} W(t) x\right|_{t=0}\right)
$$

with maximal domain. Generation of a $C$-regularized semigroup corresponds to (ACP) having a unique mild solution $u(t, x)=C^{-1} W(t) x$ for $x \in \operatorname{Im}(C)$, and the existence of a space continuously embedded between $[\operatorname{Im}(C)]$ and $X$ on which the restriction of $A$ generates a strongly continuous semigroup. Generation of an $n$-times integrated semigroup corresponds to generation of a $(\lambda-A)^{-n}$-regularized semigroup. An $I$-regularized semigroup is a strongly continuous semigroup. See [d3] for basic information on regularized semigroups.

Definition 2.3. Suppose $-A$ generates a uniformly bounded strongly continuous semigroup $\left\{e^{-t A}\right\}_{t \geq 0}$. Let $\mathcal{F}$ be the Banach algebra of functions $f$ for which there exists a complex-valued measure $\mu$ of bounded variation on $[0, \infty)$ such that

$$
f(s)=\int_{0}^{\infty} e^{-s t} d \mu(t) \quad(s \geq 0)
$$

with norm

$$
\|f\|_{\mathcal{F}} \equiv \int_{0}^{\infty} d|\mu|(t)
$$

the total variation of $\mu$.

Then the bounded operator $f(A)$ (see $[\mathrm{H}-\mathrm{P}$, Chapter XV]) is defined by

$$
f(A) x \equiv \int_{0}^{\infty}\left(e^{-t A} x\right) d \mu(t) \quad(x \in X) .
$$

The fact that $f \mapsto f(A)$ is an algebra homomorphism is equivalent to the following, from [H-P, Theorem 15.2.1]:

$$
\int_{0}^{\infty}\left(e^{t A} x\right) d\left(\mu_{1} * \mu_{2}\right)(t)=\int_{0}^{\infty} e^{r A}\left(\int_{0}^{\infty} e^{t A} x d \mu_{1}(t)\right) d \mu_{2}(r),
$$

for $\mu_{1}, \mu_{2}$ complex-valued measures of bounded variation on $[0, \infty)$ and $x \in X$.

Denote by $\delta_{0}$ the Dirac delta function, the measure such that

$$
\int_{0}^{\infty} e^{-s t} d \delta_{0}(t)=1 \quad(s \geq 0)
$$

Definition 2.6 ([d4, Definition 2.4]). Let $\operatorname{EXT}(\mathcal{F})$ (for "extension of $\mathcal{F}$ ") be the set of all complex-valued functions $g$ on $[0, \infty)$ for which there exists $h \in \mathcal{F}$ such that $h(A)$ is injective whenever $-A$ generates a uniformly 
bounded strongly continuous semigroup and

$$
\tau \mapsto h_{\tau} \equiv e^{\tau g} h
$$

is a continuous map from $[0, \infty)$ into $\mathcal{F}$.

Lemma 2.7 ([d4, Lemma 2.6]). Suppose $-A$ generates a uniformly bounded, strongly continuous semigroup. Then $\left\{h_{\tau}(A)\right\}_{\tau \geq 0}$, from Definition 2.6, is an $h(A)$-regularized semigroup that is continuous in the operator norm.

Definition 2.8 ([d4, Definition 2.7]). For $g, h, h_{\tau}$ as in Definition 2.6, denote by $g(A)$ the generator of $\left\{h_{\tau}(A)\right\}_{\tau \geq 0}$.

In the following, for $\lambda$ complex, let $g_{\lambda}(s) \equiv(\lambda-s)^{-1}, f_{0}(s) \equiv 1, f_{1}(s) \equiv s$.

Lemma 2.9 ([d4, Theorems 2.8 and 3.7 and Proposition 3.6]). If $-A$ generates a uniformly bounded, strongly continuous semigroup, then $g \mapsto g(A)$, defined by Definition 2.8, is an extension of the functional calculus (2.4) such that

(a) $f_{0}(A)=I$

(b) $f_{1}(A)=A$;

(c) $g_{\lambda}(A)=(\lambda-A)^{-1}$ whenever $\operatorname{Re}(\lambda)<0$;

(d) if $f$ and $g$ are in $\operatorname{EXT}(\mathcal{F})$, then $f g \in \operatorname{EXT}(\mathcal{F})$, with

$$
f(A) g(A) \subseteq(f g)(A), \quad \mathcal{D}(f(A) g(A))=\mathcal{D}(f g)(A) \cap \mathcal{D} g(A) .
$$

Definition 2.10. Next we describe a special case of an extension of a holomorphic Riesz-Dunford sort of functional calculus (see [d2]).

Fix $\omega>0$, and assume that the right half-plane $-\omega+\operatorname{RHP} \equiv\{z \in \mathbb{C} \mid$ $\operatorname{Re}(z)>-\omega\} \subseteq \varrho(A)$, with $\left\|(\lambda-A)^{-1}\right\|$ bounded on $-\varepsilon+$ RHP for any $\varepsilon<\omega$.

For $\varepsilon>0$, denote by $-\varepsilon+$ LHP the set of complex $z$ such that $\operatorname{Re}(z)<-\varepsilon$, and by $\mathcal{H}_{L}(-\varepsilon+$ LHP $)$ the set of complex-valued holomorphic functions $f$ on $-\varepsilon+$ LHP such that $\{\operatorname{Re}(f(z)) \mid \operatorname{Re}(z)<-\varepsilon\}$ is bounded above.

Lemma 2.11 ([d2, Theorem 3.5]). Suppose A, $\omega$, and $\varepsilon$ are as in Definition 2.10, $f \in \mathcal{H}_{L}(-\varepsilon+$ LHP $)$, and $\varepsilon<\delta<\omega$. Then

$$
W_{f}(\tau) \equiv \int_{-\delta+i \mathbb{R}} e^{\tau f(w)}(w-A)^{-1} \frac{d w}{2 \pi i w^{2}} \quad(\tau \geq 0)
$$

defines an $A^{-2}$-regularized semigroup that is continuous in the operator norm.

Definition 2.12. For $A$ as in Definition 2.10, $f(A)$ is defined to be the generator of $\left\{W_{f}(\tau)\right\}_{\tau \geq 0}$ from Lemma 2.11.

In the following, $f_{0}, f_{1}$, and $g_{\lambda}$ are as in Lemma 2.9. 
Lemma 2.13 ([d2, Theorem 3.10]). If $A$ is as in Definition 2.10, then $f \mapsto f(A)$, from Definition 2.12, has the following properties:

(a) $f_{0}(A)=I$

(b) $f_{1}(A)=A$;

(c) $g_{\lambda}(A)=(\lambda-A)^{-1}$ whenever $\operatorname{Re}(\lambda)>-\varepsilon$.

III. Behavior of the semigroup generated by $A^{-1}$ on subspaces. Throughout this section, $A$ is an injective operator. It is known ([Z1], [Gom$\mathrm{Z}-\mathrm{T}]$, and $[\mathrm{K}]$ ) that, even when the image of $A$ is dense and $A$ generates a uniformly bounded, strongly continuous semigroup, $A^{-1}$ might not generate a strongly continuous semigroup. In this section we show that, when $A$ generates a uniformly bounded, strongly continuous semigroup, then $A^{-1}$ generates an $O(1+\tau) A(1-A)^{-1}$-regularized semigroup $\{W(\tau)\}_{\tau \geq 0}$ (Theorem 3.3), so that

$$
e^{\tau A^{-1}} \equiv(1-A) A^{-1} W(\tau)
$$

is defined as a possibly unbounded closed operator for $\tau \geq 0$; we will henceforth denote $W(\tau)$ by

$$
e^{\tau A^{-1}} A(1-A)^{-1} \text {. }
$$

This means, in particular, that $\left\|e^{\tau A^{-1}} x\right\|=O(1+\tau)$ for all $x \in \operatorname{Im}(A)$, and if $0 \in \varrho(A)$, then $\left\|e^{\tau A^{-1}}\right\|=O(\tau)$ as $\tau$ goes to infinity (Corollary 3.7). We also show in this section that, if $A$ generates an exponentially stable strongly continuous semigroup, then for $k \in \mathbb{N}$ and $x \in \mathcal{D}\left(A^{k}\right),\left\|e^{\tau A^{-1}} x\right\|=$ $O\left(\tau^{1 / 4-k / 2}\right)$ as $\tau \rightarrow \infty$ (Theorem 3.9). Under the weaker hypothesis of the resolvents of $A$ being bounded in a half-plane $\operatorname{Re}(z)>-c$ for some $c>0$, for $k \in \mathbb{N},\left\|e^{\tau A^{-1}} x\right\|=O\left(\tau^{-r}\right)$ for $r<\frac{1}{2}(k-1)$ (Propositions 3.10 and 3.11).

Here is a summary of material about Bessel functions needed (see [W]). For $n$ a nonnegative integer, denote by $J_{n}$ the Bessel function of the first kind of the $n$th order. We will denote by $G$ (called $h_{a c}$ in [Z1]) the function whose Laplace transform is $1-e^{-1 / s}$ :

$$
G(t) \equiv \frac{1}{\sqrt{t}} J_{1}(2 \sqrt{t}) .
$$

Then

(1) $J_{n}(r)=O\left(r^{-1 / 2}\right)$ as $r \rightarrow \infty$ for all nonnegative integers $n$;

(2) $G$ is continuous on $(0, \infty)$ and $\lim _{t \rightarrow 0^{+}} G(t)=1$;

(3) $\int_{0}^{\infty}\left|G^{\prime}(t)\right| d t<\infty$

(4) $\left|G^{\prime}(t)\right|=O\left(t^{-5 / 4}\right)$ as $t \rightarrow \infty$;

(5) $(-d / d t)^{n} J_{0}(2 \sqrt{t})=(1 / \sqrt{t})^{n} J_{n}(2 \sqrt{t})$ for all nonnegative integers $n$;

(6) $J_{0}(0)=1$;

(7) $J_{0}(2 \sqrt{t \tau})=(d / d \tau)(\tau G(t \tau))$. 
We begin, as in [Z1, (10)], with the Laplace transform (1.1). Unfortunately $G$ is not in the Banach algebra $\mathcal{F}$ of Definition 2.3. Thus we need the extended functional calculus described in Definition 2.8, applied to $-A$.

First a technical lemma, where we use the informal notation $h(x)$ for a function $h$ of $x$.

Lemma 3.2. For any $h(x) \in L^{1}([0, \infty))$, the map $t \mapsto t^{2} h(t x)$ is continuous from $[0, \infty)$ into $L^{1}([0, \infty))$.

Proof. For any $t \geq 0$, define $T(t): L^{1}([0, \infty)) \rightarrow L^{1}([0, \infty))$ by

$$
(T(t) h)(x) \equiv t^{2} h(t x) \quad\left(h \in L^{1}([0, \infty)), x \geq 0\right) .
$$

For $h \in L^{1}([0, \infty))$ and $t \geq 0$,

$$
\|T(t) h\|_{1}=\int_{0}^{\infty} t^{2}|h(t x)| d x=\int_{0}^{\infty} t|h(r)| d r=t\|h\|_{1},
$$

thus $\|T(t)\|$ is locally bounded in $t$. This implies that it is sufficient to prove the lemma for $h$ in a dense subspace of $L^{1}([0, \infty))$. Our choice of dense subspace will be $C_{c}^{1}([0, \infty))$, the continuously differentiable functions of compact support: for $h \in C_{c}^{1}([0, \infty))$,

$$
\lim _{s \rightarrow t}\left\|s^{2} h(s x)-t^{2} h(t x)\right\|_{1}=0
$$

by dominated convergence, since $h$ is bounded and of compact support, concluding the proof.

In the following, $\operatorname{Im}(A)$ might not be dense.

Theorem 3.3. Suppose A generates a uniformly bounded, strongly continuous semigroup $\left\{e^{t A}\right\}_{t>0}$. Then $A^{-1}$ generates an operator-norm continuous $O(1+\tau) A(1-A)^{-1}$-regularized semigroup, given by

$\left(e^{\tau A^{-1}}-I\right) A(1-A)^{-1} x=\int_{0}^{\infty}\left(e^{t A} x\right) \tau\left(G(t \tau)-G(t \tau) * e^{-t}\right) d t \quad(\tau>0, x \in X)$.

For $y \in \operatorname{Im}(A)$,

$$
\left(e^{\tau A^{-1}}-I\right) y=-\int_{0}^{\infty}\left(e^{t A} y\right) \tau G(t \tau) d t .
$$

Proof. Let $h(s) \equiv-s(1+s)^{-1}, g(s) \equiv-1 / s$, and $h_{\tau} \equiv e^{\tau g} h$ for $\tau \geq 0$. To invoke Lemma 2.7, we need to show that $\tau \mapsto h_{\tau}$ is a continuous map from $[0, \infty)$ into $\mathcal{F}$ from Definition 2.3. Equivalently, since

$$
\begin{aligned}
h_{\tau}(s)=\left((1+s)^{-1}-1\right) e^{-\tau / s} & =\left((1+s)^{-1}-1\right)+\left(1-e^{-\tau / s}\right)\left(1-(1+s)^{-1}\right) \\
& =\int_{0}^{\infty} e^{-s t} H_{\tau}(t) d t \quad(s>0),
\end{aligned}
$$


where

$$
H_{\tau}(t) \equiv\left(e^{-t}-\delta_{0}(t)\right)+\tau G(t \tau) *\left(\delta_{0}(t)-e^{-t}\right) \quad(t, \tau \geq 0),
$$

we need to show that $\tau \mapsto H_{\tau}$ is a continuous map from $[0, \infty)$ into $L^{1}([0, \infty))$.

First, for $\tau>0$, rewrite

$$
\begin{aligned}
\frac{1}{\tau}\left(\left(\delta_{0}(t)-e^{-t}\right)+H_{\tau}(t)\right) & =G(t \tau)-\int_{0}^{t} e^{-(t-s)} G(s \tau) d s \\
& =G(t \tau)-\left[\left.e^{-(t-s)} G(s \tau)\right|_{0} ^{t}-\int_{0}^{t} e^{-(t-s)} \tau G^{\prime}(s \tau) d s\right] \\
& =e^{-t}+\int_{0}^{t} e^{-(t-s)} \tau G^{\prime}(s \tau) d s
\end{aligned}
$$

Thus, for any $\tau_{1}, \tau_{2} \geq 0$,

$$
H_{\tau_{1}}(t)-H_{\tau_{2}}(t)=\left(\tau_{1}-\tau_{2}\right) e^{-t}+\int_{0}^{t} e^{-(t-s)}\left(\tau_{1}^{2} G^{\prime}\left(s \tau_{1}\right)-\tau_{2}^{2} G^{\prime}\left(s \tau_{2}\right)\right) d s
$$

so that

$$
\begin{aligned}
\left\|H_{\tau_{1}}(t)-H_{\tau_{2}}(t)\right\|_{1} & =\int_{0}^{\infty}\left|\left(\tau_{1}-\tau_{2}\right) e^{-t}+\int_{0}^{t} e^{-(t-s)}\left(\tau_{1}^{2} G^{\prime}\left(s \tau_{1}\right)-\tau_{2}^{2} G^{\prime}\left(s \tau_{2}\right)\right) d s\right| d t \\
& \leq\left|\tau_{1}-\tau_{2}\right|+\int_{0}^{\infty} \int_{0}^{t} e^{-(t-s)}\left|\tau_{1}^{2} G^{\prime}\left(s \tau_{1}\right)-\tau_{2}^{2} G^{\prime}\left(s \tau_{2}\right)\right| d s d t \\
& =\left|\tau_{1}-\tau_{2}\right|+\int_{0}^{\infty}\left(\int_{s}^{\infty} e^{-(t-s)} d t\right)\left|\tau_{1}^{2} G^{\prime}\left(s \tau_{1}\right)-\tau_{2}^{2} G^{\prime}\left(s \tau_{2}\right)\right| d s \\
& =\left|\tau_{1}-\tau_{2}\right|+\int_{0}^{\infty}\left|\tau_{1}^{2} G^{\prime}\left(s \tau_{1}\right)-\tau_{2}^{2} G^{\prime}\left(s \tau_{2}\right)\right| d s .
\end{aligned}
$$

By Lemma 3.2, since $G^{\prime} \in L^{1}([0, \infty)), \lim _{\tau_{1} \rightarrow \tau_{2}}\left\|H_{\tau_{1}}-H_{\tau_{2}}\right\|_{1}=0$ for any $\tau_{2} \geq 0$. Thus $\tau \mapsto h_{\tau}$ is a continuous map from $[0, \infty)$ into $\mathcal{F}$. This means (Lemma 2.7 and Definition 2.8) that $g(-A)$ is defined as the generator of the operator-norm continuous $h(-A)$-regularized semigroup

$$
\begin{aligned}
h_{\tau}(-A) x & =\int_{0}^{\infty}\left(e^{t A} x\right) H_{\tau}(t) d t \\
& =(1-A)^{-1} x-x+\int_{0}^{\infty}\left(e^{t A} x\right) \tau\left(G(t \tau)-G(t \tau) * e^{-t}\right) d t \\
& =A(1-A)^{-1} x+\int_{0}^{\infty}\left(e^{t A} x\right) \tau\left(G(t \tau)-G(t \tau) * e^{-t}\right) d t \quad(\tau \geq 0, x \in X) .
\end{aligned}
$$


By Lemma 2.9(c), since $h(s)=(1+s)^{-1}-1$, we have $h(-A)=(1-A)^{-1}-1=$ $A(1-A)^{-1}$. By Lemma 2.9(a), (b), and (d), $A g(-A) \subseteq I$ and $g(-A) A \subseteq I$, which implies that $g(-A)=A^{-1}$.

In summary, we have shown that $A^{-1}$ generates an operator-norm continuous $A(1-A)^{-1}$-regularized semigroup, given by the first integral expression of the statement of Theorem 3.3.

For the growth of $\left\|e^{\tau A^{-1}} A(1-A)^{-1}\right\|$, recall that

$$
\begin{aligned}
& \left\|e^{\tau A^{-1}} A(1-A)^{-1}\right\| \\
& \quad=\left\|h_{\tau}\right\|_{\mathcal{F}}=\left\|H_{\tau}\right\|_{1}=\left\|t \mapsto\left(e^{-t}-\delta_{0}(t)\right)+\tau G(t \tau) *\left(\delta_{0}(t)-e^{-t}\right)\right\|_{1} \\
& \leq 2+\tau\left\|t \mapsto G(t \tau) *\left(\delta_{0}(t)-e^{-t}\right)\right\|_{1} \\
& =2+\tau\left\|t \mapsto e^{-t}+\int_{0}^{t} e^{-(t-s)} \tau G^{\prime}(s \tau) d s\right\|_{1} \\
& \leq 2+\tau+\tau \int_{0}^{\infty} \int_{0}^{t}\left|e^{-(t-s)} \tau G^{\prime}(s \tau)\right| d s d t \\
& =2+\tau+\tau \int_{0}^{\infty}\left(\int_{s}^{\infty} e^{-(t-s)} d t\right)\left|\tau G^{\prime}(s \tau)\right| d s=2+\tau+\tau \int_{0}^{\infty}\left|\tau G^{\prime}(s \tau)\right| d s \\
& =2+\tau+\tau \int_{0}^{\infty}\left|G^{\prime}(r)\right| d r=O(1+\tau),
\end{aligned}
$$

as desired, since $\int_{0}^{\infty}\left|G^{\prime}(r)\right| d r<\infty$.

Finally, suppose $y \in \operatorname{Im}(A)$. Then there exists $x \in X$ such that $y=$ $A(1-A)^{-1} x$. Since convergence is not at first glance guaranteed by any means, let us write, for $N>0$,

$$
\begin{aligned}
& \int_{0}^{N}\left(e^{t A} y\right) G(t \tau) d t \\
& =\left.\left[\left(\int_{0}^{t} e^{s A} A(1-A)^{-1} x d s\right) G(t \tau)\right]\right|_{t=0} ^{N}-\int_{0}^{N}\left(\int_{0}^{t} e^{s A} A(1-A)^{-1} x d s\right) \tau G^{\prime}(t \tau) d t \\
& =\left.\left[\left(\int_{0}^{t} \frac{d}{d s} e^{s A}(1-A)^{-1} x d s\right) G(t \tau)\right]\right|_{t=0} ^{N}-\int_{0}^{N}\left(\int_{0}^{t} \frac{d}{d s} e^{s A}(1-A)^{-1} x d s\right) \tau G^{\prime}(t \tau) d t \\
& =\left.\left[\left(\left(e^{t A}-I\right)(1-A)^{-1} x\right) G(t \tau)\right]\right|_{t=0} ^{N}-\int_{0}^{N}\left(\left(e^{t A}-I\right)(1-A)^{-1} x\right) \tau G^{\prime}(t \tau) d t .
\end{aligned}
$$

Since $\left\{e^{t A}\right\}_{t \geq 0}$ is bounded and $G(t)=O\left(t^{-3 / 4}\right)$ and $G^{\prime}(t)=O\left(t^{-5 / 4}\right)$ for $t$ 
large, we may let $N \rightarrow \infty$ to get

$$
\begin{aligned}
\int_{0}^{\infty}\left(e^{t A} y\right) G(t \tau) d t & =\int_{0}^{\infty}\left(\left(I-e^{t A}\right)(1-A)^{-1} x\right) \tau G^{\prime}(t \tau) d t \\
& =-(1-A)^{-1} x-\int_{0}^{\infty}\left(e^{t A}(1-A)^{-1} x\right) \tau G^{\prime}(t \tau) d t .
\end{aligned}
$$

On the other hand, by (2.5), and our proof so far,

$$
\begin{aligned}
\frac{1}{\tau}\left(e^{\tau A^{-1}}-I\right) y & \\
& =\int_{0}^{\infty}\left(e^{t A} x\right) \frac{1}{\tau}\left(H_{\tau}(t)+\left(\delta_{0}(t)-e^{-t}\right)\right) d t \\
& =\int_{0}^{\infty}\left(e^{t A} x\right)\left(e^{-t}+\tau G^{\prime}(t \tau) * e^{-t}\right) d t \\
& =\left(\int_{0}^{\infty}\left(e^{t A} x\right) e^{-t} d t\right)+\left(\int_{0}^{\infty} e^{t A}\left(\int_{0}^{\infty}\left(e^{t A} x\right) e^{-t} d t\right) \tau G^{\prime}(t \tau) d t\right) \\
& =(1-A)^{-1} x+\left(\int_{0}^{\infty}\left(e^{t A}(1-A)^{-1} x\right) \tau G^{\prime}(t \tau) d t\right) .
\end{aligned}
$$

Comparing (3.4) and (3.5) gives the desired representation.

Remark 3.6. In [P-Z, Theorem 3.3] it is shown that $A^{-1}$ generates a once-integrated semigroup when $A$ is injective and generates a uniformly bounded strongly continuous semigroup. This implies ([d3, Theorem 18.3]) that $A^{-1}$ generates a $\left(1-A^{-1}\right)^{-1}=A(A-1)^{-1}$-regularized semigroup. We wished to present our direct proof of this result, both to get growth conditions on the solutions of the abstract Cauchy problem (see beginning of Section II) $u(t, x)=e^{t A^{-1}} x, x \in \operatorname{Im}(A)$, and to get the intuitively natural functional calculus representation of $e^{\tau A^{-1}} y, y \in \operatorname{Im}(A)$,

$$
\left(e^{\tau A^{-1}}-I\right) y=-\int_{0}^{\infty}\left(e^{t A} y\right) \tau G(t \tau) d t
$$

which will be central to the results in Section IV. The operator-norm continuity is also of interest.

COROLlaRY 3.7. If A generates a uniformly bounded, strongly continuous semigroup $\left\{e^{t A}\right\}_{t \geq 0}$ and $0 \in \varrho(A)$, then $\left\|e^{\tau A^{-1}}\right\|=O(\tau)$ as $\tau \rightarrow \infty$, and is given by

$$
\left(e^{\tau A^{-1}}-I\right) x=-\int_{0}^{\infty}\left(e^{t A} x\right) \tau G(t \tau) d t \quad(x \in X, \tau \geq 0) .
$$


REMARK 3.8. The representation of $e^{\tau A^{-1}}$ in Corollary 3.7 appears in [Z1, Lemma 3.2], when $\left\{e^{t A}\right\}_{t \geq 0}$ is exponentially stable. In [Z1, Theorem 3.4] it is shown that if $\left\{e^{t A}\right\}_{t \geq 0}$ is exponentially stable, then $\left\|e^{\tau A^{-1}}\right\|=O\left(\tau^{1 / 4}\right)$ as $\tau \rightarrow \infty$. [Z1, Theorem 3.3] also shows, under the same hypothesis, that $\left\|e^{\tau A^{-1}} x\right\|=O\left(\tau^{-1 / 4}\right)$ for $x \in \mathcal{D}(A)$. The following theorem shows that, for $x \in \mathcal{D}\left(A^{k}\right)$ and $k>1$, we may improve the decay estimate. We are indebted to the referee for the proof, and the improved decay rate over Proposition 3.11.

TheOREM 3.9. If A generates an exponentially stable strongly continuous semigroup $\left\{e^{t A}\right\}_{t \geq 0}$ and $k \in \mathbb{N}$, then $\left\|e^{\tau A^{-1}} x\right\|=O\left(\tau^{1 / 4-k / 2}\right)$ for all $x \in \mathcal{D}\left(A^{k}\right)$.

Proof. By (1.2) (see Theorem 3.3), and Bessel function facts (7), (5), and (1), appearing after (3.1) near the beginning of this section, in that order,

$$
\begin{aligned}
e^{\tau A^{-1} A^{-k}} & =\left(\frac{d}{d \tau}\right)^{k}\left(e^{\tau A^{-1}}\right)=\left(\frac{d}{d \tau}\right)^{k} \int_{0}^{\infty} e^{t A} \tau G(t \tau) d t \\
& =\left(\frac{d}{d \tau}\right)^{k-1} \int_{0}^{\infty} e^{t A} J_{0}(2 \sqrt{t \tau}) d t \\
& =\int_{0}^{\infty} e^{t A} t^{k-1}\left(\frac{-1}{\sqrt{t \tau}}\right)^{k-1} J_{k-1}(2 \sqrt{t \tau}) d t \\
& =O\left(\left(\frac{1}{\sqrt{\tau}}\right)^{k-1}(\sqrt{\tau})^{-1 / 2}\right)=O\left(\tau^{1 / 4-k / 2}\right),
\end{aligned}
$$

as desired.

In the remainder of this section, denote by RHP the right half-plane $\{z \in \mathbb{C} \mid \operatorname{Re}(z)>0\}$.

By the Hille-Yosida theorem, when $A$ generates an exponentially stable strongly continuous semigroup, as in Theorem 3.9, there exists $c>0$ such that all powers $(\lambda-A)^{-n}$ of the resolvent of $A$ are uniformly bounded on the right half-plane $-c+\mathrm{RHP}$. In the following two propositions, we weaken this hypothesis to only require that the resolvent $(\lambda-A)^{-1}$ be bounded; we also do not require that $\mathcal{D}(A)$ be dense, as is the case when $A$ generates a strongly continuous semigroup. This results in a weaker growth condition on $\left\|e^{\tau A^{-1}}\right\|$.

Proposition 3.10. Suppose there exists $c>0$ such that $-c+\mathrm{RHP}$ $\subseteq \varrho(A)$, with $\left\|(\lambda-A)^{-1}\right\|$ bounded on $-c+\mathrm{RHP}$. Then, for all $x \in \mathcal{D}\left(A^{2}\right)$, $\left\|e^{\tau A^{-1}} x\right\|=O(\sqrt{(\ln \tau) / \tau})=O\left(\tau^{-r}\right)$ for $r<1 / 2$ as $\tau \rightarrow \infty$. 
Proof. $A$ is as in Definition 2.10. By Lemmas 2.11 and 2.13(c), there exists $\delta>0$ such that

$$
e^{\tau A^{-1}} A^{-2}=\int_{-\delta+i \mathbb{R}} e^{\tau / w}(w-A)^{-1} \frac{d w}{2 \pi i w^{2}} \quad(\tau \geq 0),
$$

with $\left\|(w-A)^{-1}\right\|$ bounded on $-\delta+i \mathbb{R}$, so that there exists a constant $M$ such that, for $\tau \geq 0$,

$$
\begin{aligned}
\left\|e^{\tau A^{-1}} A^{-2}\right\| & \leq M \int_{\mathbb{R}}\left|e^{\frac{\tau}{-\delta+i y}}\right| \frac{d y}{|-\delta+i y|^{2}} \\
& =M \int_{\mathbb{R}} e^{\frac{-\tau \delta}{\delta^{2}+y^{2}}} \frac{d y}{\delta^{2}+y^{2}}=\frac{M}{\delta} \int_{\mathbb{R}} e^{\frac{-\tau / \delta}{1+x^{2}}} \frac{d x}{1+x^{2}},
\end{aligned}
$$

where the last equality follows from the substitution $x=y / \delta$. Thus it is sufficient to show that

$$
\int_{0}^{\infty} e^{\frac{-\tau}{1+y^{2}}} \frac{d y}{1+y^{2}}=\frac{1}{2} \int_{\mathbb{R}} e^{\frac{-\tau}{1+y^{2}}} \frac{d y}{1+y^{2}}=O\left(\sqrt{\frac{\ln \tau}{\tau}}\right)
$$

as $\tau \rightarrow \infty$.

Let $N=N(\tau) \equiv \sqrt{\tau / \ln \tau}$ for $\tau>0$, and write

$$
\begin{aligned}
\int_{0}^{\infty} e^{\frac{-\tau}{1+y^{2}}} \frac{d y}{1+y^{2}} & =\int_{0}^{N} e^{\frac{-\tau}{1+y^{2}}} \frac{d y}{1+y^{2}}+\int_{N}^{\infty} e^{\frac{-\tau}{1+y^{2}}} \frac{d y}{1+y^{2}} \leq \int_{0}^{N} e^{\frac{-\tau}{1+N^{2}}} d y+\int_{N}^{\infty} \frac{d y}{1+y^{2}} \\
& =N e^{\frac{-\tau}{1+N^{2}}}+\arctan \left(\frac{1}{N}\right)=\sqrt{\frac{\tau}{\ln \tau}} e^{-\frac{\tau \ln \tau}{\tau+\ln \tau}}+\arctan \left(\sqrt{\frac{\ln \tau}{\tau}}\right) \\
& =\frac{1}{\sqrt{\ln \tau}} \tau^{\frac{1}{2}-\frac{\tau}{\tau+\ln \tau}}+\arctan \left(\sqrt{\frac{\ln \tau}{\tau}}\right) \\
& =\frac{1}{\sqrt{\ln \tau}} \tau^{\frac{1}{2}-\frac{\tau+\ln \tau-\ln \tau}{\tau+\ln \tau}}+\arctan \left(\sqrt{\frac{\ln \tau}{\tau}}\right) \\
& =\frac{1}{\sqrt{\tau \ln \tau}} \tau^{\frac{\ln \tau}{\tau+\ln \tau}}+\arctan \left(\sqrt{\frac{\ln \tau}{\tau}}\right)
\end{aligned}
$$

since $\arctan s=O(s)$ as $s \rightarrow 0$, and a L'Hospital's rule argument shows that $\tau^{(\ln \tau) /(\tau+\ln \tau)}$ converges to 1 as $\tau \rightarrow \infty$, this concludes the proof.

Almost exactly the same argument generalizes this result to $\mathcal{D}\left(A^{k}\right)$, $k \geq 2$, in the following way.

Proposition 3.11. If $A$ is as in Proposition 3.10 and $k \geq 2$, then, for all $x \in \mathcal{D}\left(A^{k}\right),\left\|e^{\tau A^{-1}} x\right\|=O\left(\tau^{-r}\right)$ for $r<\frac{1}{2}(k-1)$ as $\tau \rightarrow \infty$. 
Proof. Without loss of generality, assume $r>0$. This is the same as the proof of Proposition 3.10, except that we write

$$
e^{\tau A^{-1}} A^{-k}=\int_{-\delta+i \mathbb{R}} e^{\tau / w}(w-A)^{-1} \frac{d w}{2 \pi i w^{k}},
$$

and estimate $\int_{0}^{\infty} e^{-\tau /\left(1+y^{2}\right)} \frac{d y}{\left(1+y^{2}\right)^{k / 2}}$ by breaking the integral into $\int_{0}^{N}+\int_{N}^{\infty}$, with $N=N(\tau) \equiv \tau^{s}, s \equiv r /(k-1)$ :

$$
\begin{aligned}
\int_{0}^{\infty} e^{\frac{-\tau}{1+y^{2}}} \frac{d y}{\left(1+y^{2}\right)^{k / 2}} & \leq \int_{0}^{N} e^{\frac{-\tau}{1+N^{2}}} d y+\int_{N}^{\infty} \frac{d y}{y^{k}}=N e^{-\frac{\tau}{1+N^{2}}}+\frac{1}{k-1} N^{1-k} \\
& =\tau^{s} e^{-\frac{\tau}{1+\tau^{2 s}}}+\frac{1}{k-1} \tau^{s(1-k)}=\tau^{s} e^{-\frac{\tau}{1+\tau^{2 s}}}+\frac{1}{k-1} \tau^{-r}
\end{aligned}
$$

we conclude by noting that, letting $m \equiv s k /(1-2 s)$, since $1-2 s>0$, we have

$$
\begin{aligned}
\tau^{s} e^{-\frac{\tau}{1+\tau^{2 s}}} & =O\left(\tau^{s}\left(\frac{\tau}{1+\tau^{2 s}}\right)^{-m}\right)=O\left(\tau^{s}\left(\tau^{1-2 s}\right)^{-m}\right) \\
& =O\left(\tau^{s(1-k)}\right)=O\left(\tau^{-r}\right) .
\end{aligned}
$$

Set $C^{\infty}(A) \equiv \bigcap_{k=0}^{\infty} \mathcal{D}\left(A^{k}\right)$.

Corollary 3.12. If $A$ is as in Proposition 3.10 and $x \in C^{\infty}(A)$, then $\tau^{n}\left\|e^{\tau A^{-1}} x\right\| \rightarrow 0$ as $\tau \rightarrow \infty$, for any $n \in \mathbb{N}$.

IV. A characterization, and some sufficient conditions, for $A^{-1}$ generating a strongly continuous semigroup. Conditions equivalent to $A^{-1}$ generating a strongly continuous semigroup are in Theorem 4.3. Sufficient conditions are given in Corollary 4.5, Theorems 4.7 and 4.13, and Proposition 4.11. Proposition 4.10 gives, for an integer $k$, a sufficient condition for $\left\|e^{\tau A^{-1}} x\right\|$ to be $O\left(\tau^{-1 / 4}\right)$ as $\tau \rightarrow \infty$ for $x \in \mathcal{D}\left(A^{k+1}\right)$. Different integral representations of $\left\{e^{\tau A^{-1}}\right\}_{\tau \geq 0}$ appear in Theorems 4.3, 4.7, and 4.13, Corollary 4.5, and Proposition 4.11.

Lemma 4.1. Suppose $C_{1}$ and $C_{2}$ are bounded, injective operators, and $A$ generates a $C_{1}$-regularized semigroup $\{W(t)\}_{t \geq 0}$ that commutes with $C_{2}$. Then

(a) $\left\{C_{2} W(t)\right\}_{t \geq 0}$ is a $C_{1} C_{2}$-regularized semigroup generated by $A$;

(b) if $t \mapsto T(t) \equiv C_{2}^{-1} W(t)$ defines a strongly continuous family of operators in $B(X)$, then $\{T(t)\}_{t \geq 0}$ is a $C_{2}^{-1} C_{1}$-regularized semigroup generated by $A$.

Proof. (a) is [d3, Proposition 3.10]. For (b), let $C \equiv C_{2}^{-1} C_{1}$. By hypothesis, $C \in B(X)$, and the injectivity of $C_{1}$ implies that $C$ is injective. For 
all $s, t \geq 0$,

$$
\begin{aligned}
C_{2}^{2} T(t) T(s) & =C_{2} W(t) T(s)=W(t) C_{2} T(s)=W(t) W(s)=C_{1} W(t+s) \\
& =C_{1} C_{2} T(t+s)=C_{2} C_{1} T(t+s) ;
\end{aligned}
$$

since $C_{2}$ is injective,

$$
C_{2} T(t) T(s)=C_{1} T(t+s), \quad \text { so that } \quad T(t) T(s)=C T(t+s) ;
$$

that is, $\{T(t)\}_{t \geq 0}$ is a $C$-regularized semigroup. Part (a) now implies that the generator of $\{T(t)\}_{t \geq 0}$ is $A$.

Lemma 4.2 ([d5, Lemma 3]). If $\varrho(A)$ is nonempty and $A$ generates a $C_{j}$-regularized semigroup, for $j=1,2$, and $C \equiv C_{1}+C_{2}$ is injective, then A generates a $C$-regularized semigroup.

Recall the function $G$, defined by (3.1).

TheOREM 4.3. Suppose A generates a uniformly bounded strongly continuous semigroup $\left\{e^{t A}\right\}_{t \geq 0}$. Then the following are equivalent.

(a) $A^{-1}$ generates a strongly continuous semigroup.

(b) $A^{-1}$ generates a $(1-A)^{-2}$-regularized semigroup.

(c) $\operatorname{Im}(A)$ is dense and there exists $k \in \mathbb{N}$ such that, for all $x \in X$,

$$
\int_{0}^{\infty}\left(e^{t A} A(1-A)^{-k} x\right) \tau G(t \tau) d t \in \mathcal{D}\left((1-A)^{k-2} A^{-1}\right),
$$

with

$$
W_{k}(\tau) x \equiv(1-A)^{k-2} A^{-1} \int_{0}^{\infty}\left(e^{t A} A(1-A)^{-k} x\right) \tau G(t \tau) d t \quad(\tau \geq 0, x \in X)
$$

defining a strongly continuous family in $B(X)$.

(d) There exists $k \in \mathbb{N}$ such that, for all $x \in X$,

$$
\int_{0}^{\infty}\left(e^{t A} A(1-A)^{-k} x\right) \tau G(t \tau) d t \in \mathcal{D}\left((1-A)^{k-2} A^{-1}\right),
$$

with $W_{k}(\tau)$ from $(\mathrm{c})$ defining a family in $B(X)$ locally bounded in $\tau$.

(e) $\operatorname{Im}(A)$ is dense and

$$
S(\tau) y \equiv \int_{0}^{\infty}\left(e^{t A} y\right) \tau G(t \tau) d t \quad(y \in \operatorname{Im}(A), \tau \geq 0)
$$

defines a family of bounded operators from $\operatorname{Im}(A)$ to $X$, locally bounded in $\tau$.

(f) $\operatorname{Im}(A)$ is dense and $S(\tau)$, from (e), leaves $\operatorname{Im}(A)$ invariant, with

$$
x \mapsto\left(1-A^{-1}\right) S(\tau) A(1-A)^{-1} x \quad(\tau \geq 0, x \in X)
$$

defining a family in $B(X)$ locally bounded in $\tau$. 
Then, for $\tau \geq 0$ and $x \in X$,

$$
\left.e^{\tau A^{-1}} x=x+\left(1-A^{-1}\right) \int_{0}^{\infty}\left(e^{t A} A(1-A)^{-1}\right) x\right) \tau G(t \tau) d t .
$$

Proof. (b) $\Rightarrow(\mathrm{a})$. By hypothesis and Theorem 3.3, A generates both a $(1-A)^{-2}$-regularized semigroup and an $A(1-A)^{-1}$-regularized semigroup. Note that

$$
A(1-A)^{-1}-(1-A)^{-2}=[A(1-A)-1](1-A)^{-2},
$$

which is in $B(X)$, with inverse

$$
-(1-A)^{2}\left(\lambda_{1}-A\right)^{-1}\left(\lambda_{2}-A\right)^{-1}, \quad \lambda_{1}=\frac{1}{2}(1+\sqrt{3} i), \lambda_{2}=\frac{1}{2}(1-\sqrt{3} i),
$$

in $B(X)$. Let $C_{1} \equiv[A(1-A)-1](1-A)^{-2}$ and $C_{2} \equiv C_{1}^{-1}$. By Lemma 4.2, $A^{-1}$ generates a $C_{1}$-regularized semigroup; then, by Lemma $4.1(\mathrm{a}), A^{-1}$ generates an $I$-regularized semigroup, which is precisely a strongly continuous semigroup.

$(\mathrm{d}) \Rightarrow(\mathrm{b})$. By Theorem 3.3 and Lemma 4.1(a),

$$
\begin{aligned}
& {\left[A(1-A)^{-3}-A(1-A)^{-1} W_{k}(\tau)\right] x} \\
& =(1-A)^{-2}\left[A(1-A)^{-1} x-(1-A)^{k-1} \int_{0}^{\infty}\left(e^{t A} A(1-A)^{-k} x\right) \tau G(t \tau) d t\right] \\
& =(1-A)^{-2}\left[A(1-A)^{-1} x-\int_{0}^{\infty}\left(e^{t A} A(1-A)^{-1} x\right) \tau G(t \tau) d t\right] \quad(x \in X)
\end{aligned}
$$

defines an $A(1-A)^{-3}$-regularized semigroup generated by $A^{-1}$. By Lemma 4.1(b) with $C_{1} \equiv A(1-A)^{-3}$ and $C_{2} \equiv A(1-A)^{-1},\left\{(1-A)^{-2}-W_{k}(\tau)\right\}_{\tau>0}$ is a $(1-A)^{-2}$-regularized semigroup generated by $A$.

$(\mathrm{c}) \Rightarrow(\mathrm{d})$. By Theorem 3.3, for any $x \in X$,

$$
\begin{aligned}
W_{k}(\tau) A & (1-A)^{-1} x \\
& \equiv(1-A)^{k-2} A^{-1} \int_{0}^{\infty}\left(e^{t A} A(1-A)^{-k}\left(A(1-A)^{-1} x\right)\right) \tau G(t \tau) d t \\
& =(1-A)^{-2} \int_{0}^{\infty}\left(e^{t A} A(1-A)^{-1} x\right) \tau G(t \tau) d t
\end{aligned}
$$

is a continuous function of $\tau$; that is, $\tau \mapsto W_{k}(\tau) y$ is continuous for all $y \in \operatorname{Im}(A)$. Since $\operatorname{Im}(A)$ is dense, and $\left\|W_{k}(\tau)\right\|$ is locally bounded in $\tau$, the same is true for all $x \in X$.

(a) $\Rightarrow(\mathrm{e})$. As $A^{-1}$ generates a strongly continuous semigroup $\left\{e^{\tau A^{-1}}\right\}_{\tau>0}$, $\operatorname{Im}(A)=\mathcal{D}\left(A^{-1}\right)$ is dense. By Theorem 3.3,

$$
S(\tau) y=y-e^{\tau A^{-1}} y \quad(y \in \operatorname{Im}(A), \tau \geq 0) .
$$


The local boundedness of this family of operators follows from $\left\{e^{\tau A^{-1}}\right\}_{\tau \geq 0}$ being a strongly continuous semigroup.

$(\mathrm{e}) \Rightarrow(\mathrm{c})$. Since $\operatorname{Im}(A)$ is dense, $\{S(\tau)\}_{\tau>0}$ extends uniquely to a family of bounded operators on $X$ locally bounded in $\tau$. Strong continuity on $X$ follows from the local boundedness and the strong continuity on the dense set $\operatorname{Im}(A)$, the latter guaranteed by Theorem 3.3. It is clear from the definition of $S(\tau)$ that it commutes with $A(1-A)^{-1}$ on $\operatorname{Im}(A)$; denseness of $\operatorname{Im}(A)$ implies the same commuting on $X$. Thus, for any $x \in X$ and $\tau \geq 0$,

$$
\begin{aligned}
\int_{0}^{\infty}\left(e^{t A} A(1-A)^{-3} x\right) \tau G(t \tau) d t & =S(\tau) A(1-A)^{-3} x \\
& =A(1-A)^{-1}\left(S(\tau)(1-A)^{-2} x\right) \in \mathcal{D}\left((1-A) A^{-1}\right),
\end{aligned}
$$

with

$$
(1-A) A^{-1} \int_{0}^{\infty}\left(e^{t A} A(1-A)^{-3} x\right) \tau G(t \tau) d t=S(\tau)(1-A)^{-2} x,
$$

a family in $B(X)$ that is locally bounded in $\tau$, giving (c), with $k=3$.

$(\mathrm{a}) \Rightarrow(\mathrm{f})$. Since $A^{-1}$ generates a strongly continuous semigroup, $\operatorname{Im}(A)=$ $\mathcal{D}\left(A^{-1}\right)$ is dense. By Theorem 3.3, for $x \in X$ and $\tau \geq 0$,

$$
\begin{aligned}
\left(1-A^{-1}\right)^{-1}\left(e^{\tau A^{-1}} x-x\right) & =-A(1-A)^{-1}\left(e^{\tau A^{-1}} x-x\right) \\
& =-\left(e^{\tau A^{-1}}-I\right) A(1-A)^{-1} x \\
& =\int_{0}^{\infty}\left(e^{t A} A(1-A)^{-1} x\right) \tau G(t \tau) d t,
\end{aligned}
$$

thus $\int_{0}^{\infty}\left(e^{t A} A(1-A)^{-1} x\right) \tau G(t \tau) d t \in \mathcal{D}\left(A^{-1}\right)=\operatorname{Im}(A)$, with

$$
\left(1-A^{-1}\right) \int_{0}^{\infty}\left(e^{t A} A(1-A)^{-1} x\right) \tau G(t \tau) d t=e^{\tau A^{-1}} x-x,
$$

a family in $B(X)$ locally bounded in $\tau$.

$(\mathrm{f}) \Rightarrow(\mathrm{e})$. If $y \in \operatorname{Im}(A)$, then, for $\tau \geq 0$, since $e^{t A}\left(1-A^{-1}\right) y=$ $\left(1-A^{-1}\right) e^{t A} y$ for all $t \geq 0$,

$$
\begin{aligned}
S(\tau) y & =S(\tau)\left(1-A^{-1}\right)^{-1}\left(1-A^{-1}\right) y=-S(\tau)\left(1-A^{-1}\right) A(1-A)^{-1} y \\
& =-\left(1-A^{-1}\right) S(\tau) A(1-A)^{-1} y,
\end{aligned}
$$

so that (e) clearly follows from (f).

The integral representation of $e^{\tau A^{-1}}$ follows from the proof of (a) $\Rightarrow(\mathrm{f})$.

EXAMPLE 4.4. We will use Theorem 4.3 to show that a restriction of $-d / d x$ on $L^{1}([0, \infty))$ is an injective generator, with dense image, of a uniformly bounded strongly continuous semigroup whose inverse does not gen- 
erate a strongly continuous semigroup. See [Z1, Example 3.7] and [Gom-Z-T] for different examples.

Our choice of example is motivated by the fact that differentiation on $L^{1}([0, \infty))$ is a touchstone for generators of uniformly bounded, strongly continuous semigroups, in the sense that many properties will hold for all generators of uniformly bounded, strongly continuous semigroups if and only if they hold for this differentiation operator; see $[\mathrm{B}-\mathrm{K}-\mathrm{M}]$ for a formal treatment of this idea in the multiparameter case and $[\mathrm{B}-\mathrm{K}]$ for the one-dimensional case.

Let $B$ be the generator of right translation, the uniformly bounded strongly continuous semigroup on $L^{1}([0, \infty))$

$$
\left(e^{t B} g\right)(x) \equiv g(x-t) \quad\left(x, t \geq 0, g \in L^{1}([0, \infty))\right),
$$

where $g(r) \equiv 0$ when $r<0$. Let $A$ be the restriction of $B$ to $X \equiv \overline{\operatorname{Im}(B)}$; that is,

$$
\begin{aligned}
\mathcal{D}(A) & \equiv\{g \in X \cap \mathcal{D}(B) \mid B g \in X\}=\overline{\operatorname{Im}(B)} \cap \mathcal{D}(B), \\
A g & \equiv B g \quad \text { for } g \in \mathcal{D}(A) .
\end{aligned}
$$

It is probably well known that $A$ has dense image (even though $B$ does not), but for completeness we give the brief argument as follows. If $h \in \operatorname{Im}(B)$, then there exists $g \in \mathcal{D}(B)$ such that $h=B g$. For $\lambda>0$, let

$$
g_{\lambda} \equiv(B-\lambda)^{-1} B g=B(B-\lambda)^{-1} g \in \mathcal{D}(B) \cap \operatorname{Im}(B) \subseteq \mathcal{D}(A) .
$$

Then, for $\lambda>0$,

$$
\begin{aligned}
h-A g_{\lambda} & =B g-(B-\lambda+\lambda)(B-\lambda)^{-1} B g=-\lambda(B-\lambda)^{-1} B g \\
& =\lambda(\lambda-B)^{-1}(B-\lambda+\lambda) g=\lambda\left(\lambda(\lambda-B)^{-1} g-g\right),
\end{aligned}
$$

thus $\lim _{\lambda \rightarrow 0^{+}}\left(h-A g_{\lambda}\right)=0$, since $\left\|\lambda(\lambda-B)^{-1}\right\|$ is bounded for $\lambda>0$; that is, $\operatorname{Im}(B)$, hence $X \equiv \overline{\operatorname{Im}(B)}$, is in the closure of $\operatorname{Im}(A)$.

For $f$ as in Definition 2.3, restricted, for convenience, to absolutely continuous signed measures $d \mu=F d t$ with $F \in L^{1}([0, \infty))$,

$$
f(s)=\int_{0}^{\infty} e^{-s t} F(t) d t,
$$

recall $f(-A)$ defined by $(2.4)$ :

$$
\begin{aligned}
(f(-A) g)(x) & \equiv \int_{0}^{\infty}\left[\left(e^{t A} g\right)(x)\right] F(t) d t=\int_{0}^{\infty} g(x-t) F(t) d t \\
& =\int_{0}^{x} g(x-t) F(t) d t \equiv(g * F)(x) \quad(g \in X, x \geq 0),
\end{aligned}
$$


the convolution of $g$ with $F$. Choosing a sequence of approximate identities

$$
\left\{h_{n}\right\}_{n=1}^{\infty}, \quad h_{n}(x) \equiv n h(n x), \quad \int_{0}^{\infty} h(x) d x=1, \quad h \text { nonnegative },
$$

it is not hard to see that

$$
\|f(-A)\|=\|F\|_{1} .
$$

The same calculation shows that, for $g \in \operatorname{Im}(A)$ and $G$ as in (3.1) and Theorem 4.3,

$$
\int_{0}^{\infty}\left(e^{t A} g\right) G(t) d t=g * G .
$$

To invoke (e) of Theorem 4.3, choose the approximate identity $\left\{h_{n}\right\}_{n=1}^{\infty}$ as above with $h \in \operatorname{Im}(A)$ (e.g., $\left.h(x) \equiv e^{-x}\right)$; then by Fatou's lemma,

$$
\begin{aligned}
\varliminf_{n \rightarrow \infty}\left\|\int_{0}^{\infty}\left[\left(e^{t A} h_{n}\right)(x)\right] G(t) d t\right\| & =\underline{\lim _{n \rightarrow \infty}}\left\|\left(h_{n} * G\right)(x)\right\| \\
& =\underline{\lim _{n \rightarrow \infty}} \int_{0}^{\infty}\left|\int_{0}^{x} G(x-y) h_{n}(y) d y\right| d x \\
& =\underline{\lim _{n \rightarrow \infty}} \int_{0}^{\infty}\left|\int_{0}^{n x} G(x-r / n) h(r) d r\right| d x \\
& \geq \int_{0}^{\infty} \underline{\lim _{n \rightarrow \infty}}\left|\int_{0}^{n x} G(x-r / n) h(r) d r\right| d x \\
& =\int_{0}^{\infty}\left|\int_{0}^{\infty} G(x) h(r) d r\right| d x=\int_{0}^{\infty}|G(x)| d x,
\end{aligned}
$$

since $G$ is continuous and $h \in L^{1}([0, \infty))$. Since $G$ is not in $L^{1}([0, \infty))$, and $\left\|h_{n}\right\|_{1}=1$ for all $n$, the map

$$
g \mapsto \int_{0}^{\infty}\left(e^{t A} g\right) G(t) d t \quad(g \in \operatorname{Im}(A))
$$

is an unbounded operator from $\operatorname{Im}(A)$ to $X$. By Theorem $4.3(\mathrm{e}) \Leftrightarrow(\mathrm{a}), A^{-1}$ does not generate a strongly continuous semigroup.

Corollary 4.5. Suppose A generates a uniformly bounded strongly continuous semigroup $\left\{e^{t A}\right\}_{t \geq 0}$ and

$$
T(\tau) x \equiv \int_{0}^{\infty}\left(e^{t A}(1-A)^{-2} x\right) \tau G(t \tau) d t \quad(\tau \geq 0, x \in X)
$$

defines a family of operators in $B(X)$ such that either 
(a) $\{T(\tau)\}_{\tau \geq 0}$ is strongly continuous; or

(b) $\operatorname{Im}(A)$ is dense and $\{T(\tau)\}_{\tau \geq 0}$ is locally bounded in $\tau$.

Then $A^{-1}$ generates a strongly continuous semigroup given by

$$
e^{\tau A^{-1}} x=x-\int_{0}^{\infty}\left(e^{t A} x\right) \tau G(t \tau) d t \quad(\tau \geq 0, x \in X) .
$$

If $y \in \mathcal{D}(A)$, then

$$
e^{\tau A^{-1}} y=-\int_{0}^{\infty}\left(e^{t A} A y\right) J_{0}(2 \sqrt{t \tau}) d t \quad(\tau \geq 0) .
$$

Proof. For $x \in X$ and $\tau \geq 0$,

$$
\begin{aligned}
\int_{0}^{\infty}\left(e^{t A} A(1-A)^{-3} x\right) \tau G(t \tau) d t & =A(1-A)^{-1} \int_{0}^{\infty}\left(e^{t A}(1-A)^{-2} x\right) \tau G(t \tau) d t \\
& =A(1-A)^{-1} T(\tau) x \in \mathcal{D}\left((1-A) A^{-1}\right),
\end{aligned}
$$

thus, by Theorem $4.3((\mathrm{~d}) \Leftrightarrow(\mathrm{a})$ or $(\mathrm{c}) \Leftrightarrow(\mathrm{a}), k=3), A^{-1}$ generates a strongly continuous semigroup

$$
e^{\tau A^{-1}} x=x+\left(1-A^{-1}\right) \int_{0}^{\infty}\left(e^{t A} A(1-A)^{-1} x\right) \tau G(t \tau) d t \quad(x \in X, \tau \geq 0) .
$$

By hypothesis,

$$
\begin{aligned}
e^{\tau A^{-1}}(1- & A)^{-2} y \\
& =(1-A)^{-2} y+\left(1-A^{-1}\right) \int_{0}^{\infty}\left(e^{t A} A(1-A)^{-1}(1-A)^{-2} y\right) \tau G(t \tau) d t \\
& =(1-A)^{-2} y+\left(1-A^{-1}\right) A(1-A)^{-1} \int_{0}^{\infty}\left(e^{t A}(1-A)^{-2} y\right) \tau G(t \tau) d t \\
& =(1-A)^{-2} y-\int_{0}^{\infty}\left(e^{t A}(1-A)^{-2} y\right) \tau G(t \tau) d t \quad(y \in X, \tau \geq 0) ;
\end{aligned}
$$

that is, (4.6) holds for $x \in \operatorname{Im}\left((1-A)^{-2}\right)$. Theorem 3.3 implies the same assertion for $x \in \operatorname{Im}\left(A(1-A)^{-1}\right)$. By the calculation beginning the proof of Theorem $4.3(\mathrm{~b}) \Rightarrow(\mathrm{a})$, this implies that $e^{\tau A^{-1}}$ has the desired representation for $x \in \operatorname{Im}(C), C \equiv[A(1-A)-1](1-A)^{-2}$; that is, for all $x \in X$ and $\tau \geq 0$,

$$
e^{\tau A^{-1}} C x=C x-\int_{0}^{\infty}\left(e^{t A} C x\right) \tau G(t \tau) d t .
$$

Since $C^{-1}$ is bounded and commutes with $e^{\tau A^{-1}}$, we may apply it to both 
sides, including bringing it inside the integral, to obtain the desired representation for all $x \in X$.

Finally, suppose $y \in \mathcal{D}(A)$ and $\tau \geq 0$. Then (see Bessel function facts after (3.1))

$$
\begin{aligned}
e^{\tau A^{-1}} y & =y-\lim _{N \rightarrow \infty} \int_{0}^{N}\left(e^{t A} y\right) \tau G(t \tau) d t \\
& =y+\lim _{N \rightarrow \infty} \int_{0}^{N}\left(e^{t A} y\right) \frac{d}{d t}\left(J_{0}(2 \sqrt{t \tau})\right) d t \\
& =y+\lim _{N \rightarrow \infty}\left(\left.\left[\left(e^{t A} y\right) J_{0}(2 \sqrt{t \tau})\right]\right|_{t=0} ^{N}-\int_{0}^{N}\left(e^{t A} A y\right) J_{0}(2 \sqrt{t \tau}) d t\right) .
\end{aligned}
$$

Since $\left\{e^{t A}\right\}_{t \geq 0}$ is bounded and $J_{0}(r)=O\left(r^{-1 / 2}\right)$ as $r \rightarrow \infty$, it follows that $\left.\left.\lim _{N \rightarrow \infty}\left[e^{t A} y J_{0}(2 \sqrt{t \tau})\right]\right|_{t=0} ^{N}\right]=-y$. Thus

$$
-\int_{0}^{\infty}\left(e^{t A} A y\right) J_{0}(2 \sqrt{t \tau}) d t
$$

exists, and equals $e^{\tau A^{-1}} y$.

Theorem 4.7. Suppose $\operatorname{Im}(A)$ is dense and $A$ generates a uniformly bounded strongly continuous semigroup $\left\{e^{t A}\right\}_{t \geq 0}$ such that

$$
\int_{0}^{\infty}\left\|e^{t A}(1-A)^{-2}\right\| t^{-3 / 4} d t<\infty .
$$

Then $A^{-1}$ generates a strongly continuous semigroup given by

$$
e^{\tau A^{-1}} x=x-\int_{0}^{\infty}\left(e^{t A} x\right) \tau G(t \tau) d t \quad(x \in X, \tau>0),
$$

satisfying $\left\|\left(e^{\tau A^{-1}}-I\right)(1-A)^{-2}\right\|=O\left(\tau^{1 / 4}\right)$ for $\tau \geq 0$.

Proof. Since $\|G(s)\|=O\left(s^{-3 / 4}\right)$ as $s \rightarrow \infty$ and $G$ is continuous on $[0, \infty)$, there exists a constant $M$ so that

$$
\|G(s)\| \leq M s^{-3 / 4} \quad \text { for all } s \geq 0,
$$

thus $t \mapsto e^{t A}(1-A)^{-2} \tau G(t \tau)$ is absolutely integrable on $[0, \infty)$, hence integrable; that is,

$$
\int_{0}^{\infty}\left(e^{t A}(1-A)^{-2} x\right) \tau G(t \tau) d t
$$


exists for all $x \in X$, with

$$
\begin{aligned}
\| \int_{0}^{\infty}\left(e^{t A}(1-A)^{-2} x\right) & \tau G(t \tau) d t \| \\
\leq & \int_{0}^{\infty}\left\|\left(e^{t A}(1-A)^{-2} x\right) \tau G(t \tau)\right\| d t \\
\leq & \int_{0}^{\infty}\left\|e^{t A}(1-A)^{-2}\right\|\|x\|\left(M(t / \tau)^{-3 / 4}\right) \tau d t \\
& =M\|x\| \tau^{1 / 4} \int_{0}^{\infty}\left\|e^{t A}(1-A)^{-2}\right\| t^{-3 / 4} d t=O\left(\tau^{1 / 4}\right) .
\end{aligned}
$$

This simultaneously allows us to apply Corollary 4.5 and to conclude the growth condition.

REMARKS 4.8. Both the hypotheses and the conclusions of Theorem 4.7 would be much more prohibitive without the regularization by $(1-A)^{-2}$.

First, the unregularized conclusion $\left\|e^{\tau A^{-1}}-I\right\|=O\left(\tau^{1 / 4}\right)$ would imply that $\tau \mapsto e^{\tau A^{-1}}$ is continuous in the operator norm, which would imply that $A^{-1}$ is bounded ([Pa, Theorem 1.1.2]). However, a quick mean-value theorem argument shows that if $\{T(t)\}_{t \geq 0}$ is a strongly continuous semigroup, then $t \mapsto T(t)(1-A)^{-1}$ is Lipschitz continuous in the operator norm; in fact, $\left\|(T(t)-I)(1-A)^{-1}\right\|=O(t)$.

Second, the unregularized hypothesis, even with $x$ thrown in for continuity, $\int_{0}^{\infty}\left\|e^{t A} x\right\| t^{-3 / 4} d t$ finite for all $x \in X$, would probably imply that $\left\{e^{t A}\right\}_{t \geq 0}$ is exponentially stable. This would follow from the existence of $t_{0}$ such that $\left\|e^{t_{0} A}\right\|<1$ ([N, Chap. A-IV, Proposition 1.10]), or $p \geq 1$ such that $\int_{0}^{\infty}\left\|e^{t A} x\right\|^{p} d t$ is finite for all $x \in X$ ([Pa, Theorem 4.4.1]).

The following example shows that the hypothesis of Theorem 4.7 does not imply that $\left\{e^{t A}\right\}_{t \geq 0}$ is exponentially stable. Let

$$
\begin{gathered}
\Omega \equiv\left\{z \in \mathbb{C}|-1 \leq \operatorname{Re}(z)<0,| \operatorname{Im}(z) \mid>e^{-1 / \operatorname{Re}(z)}\right\} \cup\{z \in \mathbb{C} \mid \operatorname{Re}(z)<-1\}, \\
X \equiv\left\{f \in H^{\infty}(\Omega) \cap C(\bar{\Omega}) \mid \lim _{|z| \rightarrow \infty, z \in \Omega} f(z)=0\right\},
\end{gathered}
$$

$(A f)(z) \equiv z f(z)$, with maximal domain, the generator of $\left(e^{t A} f\right)(z)=$ $e^{t z} f(z)(f \in X, z \in \Omega, t \geq 0)$. Then, writing complex $z=x+i y, x, y$ real, we have

$$
\begin{aligned}
\left\|e^{t A} A^{-2}\right\| & =\sup _{z \in \Omega}\left|e^{t z} z^{-2}\right|=\sup _{-1 \leq x<0} e^{t x}\left(x^{2}+\left(e^{-1 / x}\right)^{2}\right)^{-1} \\
& \leq \sup _{-1 \leq x<0} e^{t x}\left(\left(e^{-1 / x}\right)^{2}\right)^{-1}=\sup _{-1 \leq x<0} e^{t x+2 / x}
\end{aligned}
$$


which, for $t \geq 2$, equals $e^{-2 \sqrt{2 t}}$. In fact, it is not hard to see that

$$
\lim _{t \rightarrow \infty} e^{2 \sqrt{2 t}}\left\|e^{t A}\right\|=1
$$

Thus $\left\|e^{t A} A^{-2}\right\|$, hence $\left\|e^{t A}(1-A)^{-2}\right\|=\left\|e^{t A} A^{-2}\left(A^{2}(1-A)^{-2}\right)\right\|$, converges to zero as $t \rightarrow \infty$, and is in $L^{1}([0, \infty))$, but is not exponentially stable, hence $\left\{e^{t A}\right\}_{t \geq 0}$ is not exponentially stable.

REMARK 4.9. Theorem 4.7 should be compared to [Z1, Theorem 3.4], where it is shown that $\left\|e^{\tau A^{-1}}\right\|=O\left(\tau^{1 / 4}\right)$ as $\tau \rightarrow \infty$ when $\left\{e^{t A}\right\}_{t \geq 0}$ is exponentially stable; that is, $\left\|e^{t A}\right\|=O\left(e^{-\varepsilon t}\right)$ for some $\varepsilon>0$, which would clearly satisfy the hypothesis of Theorem 4.7. Similarly, the next result generalizes [Z1, Theorem 3.3], where it is shown that $\left\|e^{\tau A^{-1}} x\right\|=O\left(\tau^{-1 / 4}\right)$ when $x \in \mathcal{D}(A)$ and $\left\{e^{t A}\right\}_{t \geq 0}$ is exponentially stable, so that we could choose $k=0$ in the following.

Proposition 4.10. Suppose $\operatorname{Im}(A)$ is dense, $k$ is a nonnegative integer, and $A$ generates a uniformly bounded strongly continuous semigroup $\left\{e^{t A}\right\}_{t \geq 0}$ such that

$$
\int_{0}^{\infty}\left\|e^{t A}(1-A)^{-k}\right\| t^{-1 / 4} d t<\infty \quad \text { and } \quad \int_{0}^{\infty}\left\|e^{t A}(1-A)^{-2}\right\| t^{-3 / 4} d t<\infty .
$$

Then $\left\|e^{\tau A^{-1}} y\right\|=O\left(\tau^{-1 / 4}\right)$ for $y \in \mathcal{D}\left(A^{k+1}\right)$.

Proof. There exists a constant $M$ so that

$$
\left\|J_{0}(s)\right\| \leq M s^{-1 / 2} \quad \text { for all } s \geq 0
$$

thus by Corollary 4.5 and Theorem 4.7,

$$
\begin{aligned}
\| e^{\tau A^{-1}}(1 & -A)^{-(k+1)} \| \\
& =\left\|\int_{0}^{\infty}\left(e^{t A} A(1-A)^{-(k+1)}\right) J_{0}(2 \sqrt{t \tau}) d t\right\| \\
& \leq\left\|A(1-A)^{-1}\right\| \int_{0}^{\infty}\left\|e^{t A}(1-A)^{-k}\right\| M(2 \sqrt{t \tau})^{-1 / 2} d t \\
& =\left(\left\|A(1-A)^{-1}\right\| \frac{M}{\sqrt{2}}\right) \tau^{-1 / 4} \int_{0}^{\infty}\left\|e^{t A}(1-A)^{-k}\right\| t^{-1 / 4} d t=O\left(\tau^{-1 / 4}\right)
\end{aligned}
$$

for $\tau$ large.

Proposition 4.11. Suppose A generates a strongly continuous semigroup such that $\left\|\int_{0}^{t} e^{s A}(1-A)^{-2} d s\right\|=O\left(t^{r}\right)$ as $t \rightarrow \infty$, for some $r<1 / 4$. Then $A^{-1}$ generates a strongly continuous semigroup given by

$$
e^{\tau A^{-1}} x=x-\int_{0}^{\infty}\left(e^{t A} x\right) \tau G(t \tau) d t=x+\int_{0}^{\infty}\left(\int_{0}^{t} e^{s A}(1-A)^{-2} x d s\right) \tau^{2} G^{\prime}(t \tau) d t
$$

for $x \in X$ and $\tau \geq 0$. 
Proof. For $N>0, x \in X$ and $\tau \geq 0$, $N$

$$
\begin{aligned}
& \int_{0}^{N}\left(e^{t A}(1-A)^{-2} x\right) G(t \tau) d t \\
& \quad=\left.\left(\int_{0}^{t} e^{s A}(1-A)^{-2} x d s\right) G(t \tau)\right|_{t=0} ^{N}-\int_{0}^{N}\left(\int_{0}^{t} e^{s A}(1-A)^{-2} x d s\right) \tau G^{\prime}(t \tau) d t .
\end{aligned}
$$

Since $G(r)=O\left(r^{-3 / 4}\right)$ and $G^{\prime}(r)=O\left(r^{-5 / 4}\right)$ and is integrable on $[0, \infty)$, our growth condition allows us to take the limit as $N \rightarrow \infty$; we will use the notation $T(\tau)$, from Corollary 4.5:

$$
\begin{aligned}
T(\tau) x & \equiv \int_{0}^{\infty}\left(e^{t A}(1-A)^{-2} x\right) \tau G(t \tau) d t \\
& =-\int_{0}^{\infty}\left(\int_{0}^{t} e^{s A}(1-A)^{-2} x d s\right) \tau^{2} G^{\prime}(t \tau) d t .
\end{aligned}
$$

The continuity of $\tau \mapsto T(\tau) x$ on $[0, \infty)$, for $x \in X$, will follow from Lemma 3.2 , by rewriting, letting $S(t) x \equiv \int_{0}^{t} e^{s A}(1-A)^{-2} x d s$,

$$
-T(\tau) x=\int_{0}^{\infty} \frac{S(t) x}{\left(1+(t \tau)^{r}\right)} \tau^{2}\left[\left(1+(t \tau)^{r}\right) G^{\prime}(t \tau)\right] d t .
$$

By Lemma 3.2 with $h(x) \equiv\left(1+x^{r}\right) G^{\prime}(x), \tau \mapsto \tau^{2}\left(1+(t \tau)^{2}\right) G^{\prime}(t \tau)$ is a continuous map from $[0, \infty)$ into $L^{1}([0, \infty))$; this, combined with the fact that $\sup _{t \geq 0}\left\|S(t) x /\left(1+(t \tau)^{r}\right)\right\|$ is locally bounded in $\tau$, implies that $\tau \mapsto$ $T(\tau) x$ is a continuous map from $[0, \infty)$ into $X$, for any $x \in X$.

The result now follows from Corollary 4.5 and (4.12) above.

Inspection of the proof shows that, aside from Corollary 4.5, it is only $\int_{0}^{t} e^{s A} d s$, the (once) integrated semigroup generated by $A$, that is needed. In fact, using the candidate for $e^{\tau A^{-1}}$ given in Proposition 4.11, we may remove the hypothesis that $A$ generate a strongly continuous semigroup.

Theorem 4.13. Suppose that $A$ generates a once-integrated semigroup $\{S(t)\}_{t \geq 0}$ such that $\|S(t)\|=O\left(t^{r}\right)$ as $t \rightarrow \infty$ for some $r<1 / 4$. Then $A^{-1}$ generates a strongly continuous semigroup given by

$$
e^{\tau A^{-1}} x=x+\int_{0}^{\infty}(S(t) x) \tau^{2} G^{\prime}(t \tau) d t \quad(\tau \geq 0, x \in X) .
$$

Proof. Let

$$
T(\tau) x \equiv x+\int_{0}^{\infty}(S(t) x) \tau^{2} G^{\prime}(t \tau) d t \quad(\tau \geq 0, x \in X) .
$$

The continuity of $\tau \mapsto T(\tau) x$ on $[0, \infty)$ for $x \in X$ follows exactly as in the proof of Proposition 4.11. 
We will use the Laplace transform characterization of integrated semigroups (see Definition 2.1) to show that $\{T(\tau)\}_{\tau \geq 0}$ is a strongly continuous semigroup generated by $A^{-1}$. We begin again with our favorite Laplace transform (1.1), with variables changed,

$$
\int_{0}^{\infty} e^{-\lambda \tau} G(\tau) d \tau=1-e^{-1 / \lambda}
$$

then differentiate with respect to $\lambda$ :

$$
\int_{0}^{\infty} \tau e^{-\lambda \tau} G(\tau) d \tau=\frac{1}{\lambda^{2}} e^{-1 / \lambda}, \quad \int_{0}^{\infty} \tau^{2} e^{-\lambda \tau} G(\tau) d \tau=\left(\frac{2}{\lambda^{3}}-\frac{1}{\lambda^{4}}\right) e^{-1 / \lambda} .
$$

For $\lambda>0$ and $x \in X$,

$$
\begin{aligned}
\int_{0}^{\infty} e^{-\lambda \tau}(T(\tau) x) d \tau-\frac{1}{\lambda} x & =\int_{0}^{\infty} e^{-\lambda \tau}\left[\int_{0}^{\infty}(S(t) x) \tau^{2} G^{\prime}(t \tau) d t\right] d \tau \\
& =\int_{0}^{\infty}(S(t) x)\left(\int_{0}^{\infty} e^{-\lambda \tau} \tau^{2} G^{\prime}(t \tau) d \tau\right) d t \\
& =\int_{0}^{\infty}(S(t) x)\left(\int_{0}^{\infty}\left(\lambda \tau^{2}-2 \tau\right) e^{-\lambda \tau} G(t \tau) \frac{d \tau}{t}\right) d t
\end{aligned}
$$

Applying the Laplace transforms in (4.14) to the inner integral gives

so that

$$
\begin{aligned}
\int_{0}^{\infty}\left(\lambda \tau^{2}\right. & -2 \tau) e^{-\lambda \tau} G(t \tau) \frac{d \tau}{t}=\int_{0}^{\infty}\left(\lambda \frac{\tau^{2}}{t^{2}}-2 \frac{\tau}{t}\right) e^{-\lambda \tau / t} G(\tau) \frac{d \tau}{t^{2}} \\
& =\frac{\lambda}{t^{4}} \int_{0}^{\infty} \tau^{2} e^{-\lambda \tau / t} G(\tau) d \tau-\frac{2}{t^{3}} \int_{0}^{\infty} \tau e^{-\lambda \tau / t} G(\tau) d \tau \\
& =\frac{\lambda}{t^{4}} e^{-t / \lambda}\left(2\left(\frac{t}{\lambda}\right)^{3}-\left(\frac{t}{\lambda}\right)^{4}\right)-\frac{2}{t^{3}} e^{-t / \lambda}\left(\frac{t}{\lambda}\right)^{2}=-\frac{1}{\lambda^{3}} e^{-t / \lambda}
\end{aligned}
$$

$$
\begin{aligned}
\int_{0}^{\infty} e^{-\lambda \tau}( & T(\tau) x) d \tau \\
= & \frac{1}{\lambda} x-\frac{1}{\lambda^{3}} \int_{0}^{\infty} e^{-t / \lambda}(S(t) x) d t=\frac{1}{\lambda} x-\frac{1}{\lambda^{3}}\left(\lambda\left(\frac{1}{\lambda}-A\right)^{-1}\right) x \\
= & \frac{1}{\lambda} x-\frac{1}{\lambda^{2}}\left(\frac{1}{\lambda} A\left(A^{-1}-\lambda\right)\right)^{-1} x=\frac{1}{\lambda} x-\frac{1}{\lambda^{2}} \lambda A^{-1}\left(A^{-1}-\lambda\right)^{-1} x \\
= & \frac{1}{\lambda} x+\frac{1}{\lambda} A^{-1}\left(\lambda-A^{-1}\right)^{-1} x=\frac{1}{\lambda}\left[1+\left(A^{-1}-\lambda+\lambda\right)\left(\lambda-A^{-1}\right)^{-1}\right] x \\
= & \left(\lambda-A^{-1}\right)^{-1} x,
\end{aligned}
$$

as desired. 
REMARK 4.15. If $A$ generates a once-integrated semigroup that is bounded, it can be shown that $0 \in \varrho(A)$. On the other hand, if $A$ generates a strongly continuous semigroup $e^{t A}$ that is bounded, then the once-integrated semigroup $\int_{0}^{t} e^{s A} d s$ is $O(t)$. The $O\left(t^{r}\right), r<1 / 4$, for $t$ large, behavior of Theorem 4.13 is between these two extremes.

\section{Open questions}

1. Is the growth rate of $\left\|e^{\tau A^{-1}}\right\|$ from Theorem 3.3 optimal? It can be shown that $t \mapsto \tau^{-1 / 4} H_{\tau}(t)$ ( $H_{\tau}$ from the proof of Theorem 3.3) is pointwise bounded as $\tau$ goes to infinity; the growth condition $\left\|e^{\tau A^{-1}}\right\|=O\left(1+\tau^{1 / 4}\right)$, which holds when $e^{t A}$ is exponentially stable ([Z1, Theorem 3.4]), is equivalent to $\left\|t \mapsto \tau^{-1 / 4} H_{\tau}(t)\right\|_{1}$ being bounded as $\tau$ goes to infinity.

2. Does $A^{-1}$ generate an $A^{r}(1-A)^{-r}$-regularized semigroup, for $0<r$ $<1$, when $A$ generates a uniformly bounded strongly continuous semigroup? This would bring us closer to $A^{-1}$ generating a strongly continuous semigroup, producing solutions of the abstract Cauchy problem (ACP), with $A$ replaced by $A^{-1}$, for initial data in $\operatorname{Im}\left(A^{r}\right)$.

This question could be attacked similarly to the proof of Theorem 3.3, if one had manageable expressions for the inverse Laplace transforms of $s \mapsto s^{r}(1+s)^{-r}$, call them $F_{r}$; that is,

$$
s^{r}(1+s)^{-r}=\int_{0}^{\infty} e^{-s t} F_{r}(t) d t \quad(s \geq 0) .
$$

The goal would then be to show that $G * F_{r}$ is in $L^{1}([0, \infty))$, with $\tau \mapsto$ $\tau G(t \tau) * F_{r}(t)$ a continuous map from $[0, \infty)$ into $L^{1}([0, \infty))$.

3. Does the converse of Corollary 4.5 hold?

4. As mentioned in the Introduction, Theorems 3.9 and 4.13 suggest a possible relationship between (not necessarily strongly continuous) semigroups generated by $A$ and $A^{-1}$, that regularity of $e^{t A}$ might be equivalent to decay of $e^{\tau A^{-1}}$ (hence the converse). Can this be made precise? More generally, as in (4) of the Introduction, what is the relationship between $e^{t A}$ and $e^{\tau A^{-1}}$ ?

Acknowledgments. The author greatly appreciates the thorough refereeing of this paper. In particular, the referee simplified the proofs of Lemma 3.2 and Theorem 3.9, and improved the decay rate in Theorem 3.9 from that of Proposition 3.11.

The author is indebted to Hans Zwart, A. M. Gomilko, and Sergey Piskarev for sending interesting reprints and preprints on this subject. 


\section{References}

[A] W. Arendt, Ch. Batty, M. Hieber, and F. Neubrander, Vector-Valued Laplace Transforms and Cauchy Problems, Monogr. Math. 96, Birkhäuser, 2001.

[B-K] B. Baeumer and M. Kovács, Subordinated groups of linear operators: properties via the transference principle and the related unbounded operational calculus, preprint.

[B-K-M] B. Baeumer, M. Kovács, and M. M. Meerschaert, Subordinated multiparameter groups of linear operators: properties via the transference principle, in: Functional Analysis and Evolution Equations: Dedicated to Gunter Lumer, H. Amann et al. (eds.), Birkhäuser, 2008, 35-50.

[d1] R. deLaubenfels, Inverses of generators, Proc. Amer. Math. Soc. 104 (1988), 443-448.

[d2] - Unbounded holomorphic functional calculus and abstract Cauchy problems for operators with polynomially bounded resolvent, J. Funct. Anal. 114 (1993), 348-394.

[d3] - Existence Families, Functional Calculi, and Evolution Equations, Lecture Notes in Math. 1570, Springer, 1994.

[d4] - Automatic extensions of functional calculi, Studia Math. 114 (1995), $237-259$.

[d5] - Inverses of generators of integrated or regularized semigroups, Semigroup Forum 75 (2007), 475-463.

[D] G. Doetsch, Introduction to the Theory and Application of the Laplace Transformation, Springer, Berlin, 1970.

[E-N] K.-J. Engel and R. Nagel, One-Parameter Semigroups for Linear Evolution Equations, Grad. Texts in Math. 194, Springer, 2000.

[Gol] J. A. Goldstein, Semigroups of Linear Operators and Applications, Oxford Univ. Press, New York, 1985.

[Gom1] A. M. Gomilko, The Cayley transform of the generator associated with a uniformly bounded $C_{0}$-semigroup of operators, Ukrain. Mat. Zh. 56 (2004), 1018-1029 (in Russian); English transl.: Ukrainian Math. J. 56 (2004), $1212-1226$.

[Gom2] - On the inverse of the generator of a bounded $C_{0}$-semigroup, Funktsional. Anal. Prilozhen. 38 (2004), no. 4, 6-12 (in Russian); English transl.: Funct. Anal. Appl. 38 (2004), 243-248.

[Gom-Z] A. Gomilko and H. Zwart, The Cayley transform of the generator of a bounded $C_{0}$ semigroup, Semigroup Forum 74 (2007), 140-148.

[Gom-Z-T] A. Gomilko, H. Zwart, and Y. Tomilov, On the inverse of the generator of $C_{0}$ semigroup, Mat. Sb. 198 (2007), no. 8, 35-50 (in Russian); English transl.: Sb. Math. 198 (2007), 1095-1110.

[Gor-M] M. L. Gorbachuk and I. T. Matsishin, Behavior at infinity of solutions of a first-order differential equation of parabolic type in a Banach space, Dokl. Akad. Nauk SSSR 312 (1990), 521-524 (in Russian); English transl.: Soviet Math. Dokl. 41 (1990), 442-445.

[H-P] E. Hille and R. S. Phillips, Functional Analysis and Semigroups, Amer. Math. Soc. Colloq. Publ. 31, Amer. Math. Soc., 1957.

[K] H. Komatsu, Fractional powers of operators, Pacific J. Math. 19 (1966), 285-346.

[N] R. Nagel (ed.), One-Parameter Semigroups of Positive Operators, Lecture Notes in Math. 1184, Springer, 1986. 
[P-Z $\quad$ S. Piskarev and H. Zwart, Crank-Nicolson scheme for abstract linear systems, Numer. Funct. Anal. Optim. 28 (2007), 717-736.

[Pa] A. Pazy, Semigroups of Linear Operators and Applications to Partial Differential Equations, Appl. Math. Sci. 44, Springer, 1983.

[vC] J. A. van Casteren, Generators of Strongly Continuous Semigroups, Res. Notes Math. 115, Pitman, 1985.

[W] G. N. Watson, Theory of Bessel Functions, 2nd ed., Cambridge Univ. Press, 1962.

[Z1] H. Zwart, Is $A^{-1}$ an infinitesimal generator?, in: Perspectives in Operator Theory, Banach Center Publ. 75, Inst. Math., Polish Acad. Sci., 2007, 303313.

[Z2] - Growth estimates for $\exp \left(A^{-1} t\right)$ on a Hilbert space, Semigroup Forum 74 (2007), 487-494.

1841 Drew Avenue, Columbus, OH 43235, U.S.A.

Current address:

E-mail: exmathprof@yahoo.com

Department of Mathematics

Ohio State University

Columbus, OH 43210, U.S.A.

Received September 23, 2007

Revised version August 17, 2008 